\title{
The IMIA Strategic Plan - Towards IMIA 2015
}

\section{P.J. Murray (Interim Vice President Strategic Planning Implementation, International Medical Informatics Association)}

Nocton, Lincolnshire, UK

\section{Introduction}

'Towards IMIA2015 - the IMIA Strategic Plan', was unanimously approved and adopted by the IMIA General Assembly, its governing body, when it met on August 18, 2007 prior to Medinfo2007 in Brisbane, Australia. This marked the culmination of several years' work by the IMIA Strategic Planning Task force, and of a process that sought to fully engage the membership of IMIA in the development of the IMIA Strategic Plan.

This is the first time that IMIA has had a Strategic Plan in its 40 year history, a move that represents a significant shift in thinking and planning. After the establishment in 1967 of Technical Committee 4 of IFIP (International Federation for Information Processing), under the guidance of Dr. François Grémy (France), and following a period of growth and evolution, in 1979 the International Medical Informatics Association (IMIA) was set up as an independent organization incorporated under Swiss law. IMIA has a 40 year history of significant accomplishments and, like many other organizations, has been shaped by its past members and leaders, many of whom have demonstrated remarkable commitment of time and energies to IMIA. IMIA today has approximately 150 organizational members, comprising national and affiliate members, regions, correspondents, corporations, academic institutions, special interest and working groups; and some colleagues who have been present since the creation of IMIA still actively contribute to the organization. The process of developing the IMIA Strategic Plan has been discussed elsewhere $[1,2,3]$ and will not be repeated in detail. However, it is important to note that many people have contributed to the evolution of this document; the IMIA Strategic Planning Task force interacted electronically and met at several IMIA General Assemblies, while General Assembly members contributed in 2005 (Geneva) and 2006 (Washington, DC). To ensure acceptance and ownership of the Strategic Plan at all levels of the organization, the IMIA Board, meeting in Nashville, Tennessee, USA in early 2007 contributed several significant revisions to the draft Strategic Plan, resulting in the final version adopted in August 2007. The IMIA Strategic Planning Task Force comprised Nancy Lorenzi (USA), President and Chair; Floyd Eisenberg (USA); HM Goh (Malaysia); Steven Huesing (Canada); Fernando Martin-Sanchez (Spain); Lincoln de Assis Moura, Jr. (Brazil); Peter Murray (UK); Heather Strachan (UK). Between them, they represented all parts of the 'IMIA family', i.e. national member societies, working and special interest groups, corporations, academic members and IMIA headquarters. While their main work is now complete, they will maintain an advisory role to the new Vice President for Strategic Planning Implementation in the early phases of developing and implementing the 'Transition Plan'. 
As a new generation of professionals becomes involved with IMIA, it is important to plan to ensure that we continue to be a vital organization, representing the entire spectrum of health and biomedical informatics world wide. We need to bring together the ideas of people who have grown up in an increasingly digital age, where rapid communications and global interactions are second nature, and the wisdom and experience of those who have steered IMIA through its first 40 years. IMIA is now turning attention to the implementation of the IMIA Strategic Plan, with the motto 'let a thousand flowers bloom' signifying the wide range of activities and interactions we wish to encourage.

IMIA intends to work towards turning the vision into reality. A key first step in the 'Transition Plan' is to ensure that the IMIA Strategic Plan is widely disseminated, within IMIA and to the wider global health and biomedical informatics communities. There follows a slightly edited version of the Strategic Plan, to fit the constraints of printbased publication; the full document is accessible on the IMIA website (http:// www.imia.org ) [4] We invite all members of the IMIA 'family', and all with interest in, and links to, our work to become involved in ensuring the successful implementation of the IMIA Strategic Plan.

\section{Towards IMIA 2015 - The IMIA Strategic Plan}

\section{Vision}

There will be a world-wide systems approach for healthcare. Clinicians, researchers, patients and people in general will be supported by informatics tools, processes and behaviours that make it easy to do the right thing, in the right way, at the right time to improve health care for all. This systems approach will incorporate and integrate research, clinical care and public health. To achieve this vision will require everyone being supported by informatics-based information and communication systems and technologies.

IMIA will provide leadership and expertise to the multidisciplinary health focused community and policy makers to enable the transformation of healthcare in accord with the worldwide vision to improve the health of the world population. IMIA will fulfil its vision by:

- being the scientific informatics association through which the world's knowledge leaders come together to effectively and efficiently create, assemble, integrate, synthesize or assimilate intellectual knowledge that is required worldwide to advance biomedical informatics in its role of improving health and healthcare.

- being the informatics association that effectively and efficiently connects people and the nations the world to be able to accomplish the above purpose.

(Here, 'biomedical informatics' is used in the broadest meaning and includes all the possible informatics modifiers, such as health, medical, nursing, bio and others.)

Organizations with "serious transformational intent see technology as a strategic asset and not just a tactical tool." [5] Inherent in IMIA's role is to bring together, from a global perspective, scientists, researchers, users, vendors, developers, consultants and suppliers in an environment of cooperation and sharing to research and develop the concepts needed to support the organizations of the world, and seeing technology as transformational.
As an organization committed to promoting best practice in the use of information and communication technologies within biomedical informatics and in health and healthcare, IMIA will ensure that it uses and promotes best practice in its own use of technology as a transformational strategic asset.

\section{IMIA's Conceptual Framework IMIA's Guiding Principles}

The IMIA Strategic Plan is based on three guiding principles, which are:

1. IMIA will help to ensure a holistic, systems approach to collaboration and networking about biomedical informatics to maximize impact world-wide.

2. IMIA will uphold an ethical approach to informatics systems as identified by IMIA's seven ethical principles. [6]

3. IMIA will promote biomedical informatics based on evidence and best practice to ensure impact on health is high quality, safe, efficient and sustainable and that makes it easy to do the right thing, in the right way, at the right time worldwide.

\section{IMIA's Integrated Strategic Framework Model}

IMIA as an organization and through it members has numerous possibilities for integration and connections. The Strategic Planning Task Force evolved a concentric circle model to conceptualize and demonstrate the potential vastness of IMIA's connections and integration possibilities. The Strategic Planning Task Force thought of this model as a colourful umbrella. 


\section{Five Concentric Circles:}

The IMIA "umbrella" starts with concentric circles to illustrate IMIA's multiple dimensions. (Figure 1)

- The core dimension of IMIA is knowledge. Knowledge is represented as the central core from which all other circles as well as IMIA's strategies, interactions and efforts emanate. Knowledge means the concepts, methods, education, and research that underpin health and biomedical informatics directly and indirectly.

- The second circle (from the knowledge core) represents the science layer. Within this circle IMIA members have their connection and integration with the science and discovery of informatics.

- The third circle represents the application layer of the scientific discoveries. Within this circle are the informatics applications that address the multiple practical applications of informatics tools.

- The fourth circle represents IMIA's impact layer. This refers to the potential impact that IMIA can have on governments, nations, outcomes, health professionals, individuals and others.

- The fifth (or outer) circle represents the people layer. Items in this circle include individuals, citizen organizations, personal health involvement, dissemination and acceptance, enabling personal responsibility, and public/personal health.

\section{The Six "Pie" Sectors}

Another planning dimension outlines key subject-focused sectors. Superimposed on the five concentric circle layers are six major sectors. Knowledge is also the core of each of the six sectors. (Figure 2)

- The sectors start at the top of the circle with the health sector. The components within the health sec-

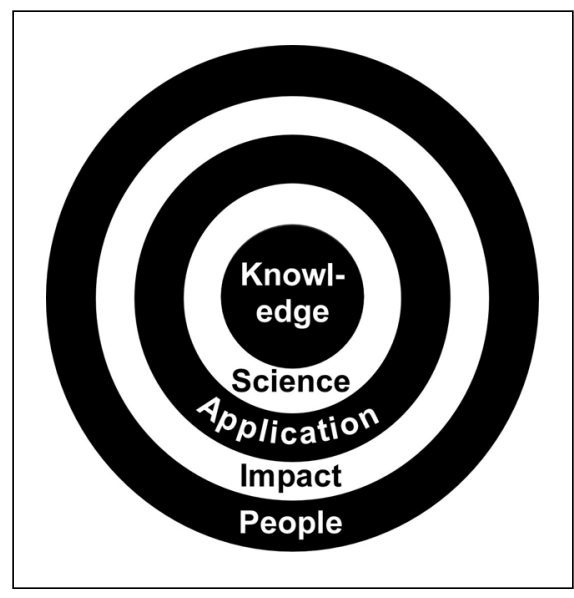

Fig. 1 The five circles ofthe IMIA Integrated Strategic Planning Framework

tor represent the IMIA vision. The components are: patho-physiology (science layer); diagnosis, therapy, treatment (application layer); public health and health improvement (impact layer) and personal health (people layer).

- The next sector is research (how we understand and create to support health). The components within this sector include: basic core research (science layer); applied science (application layer); evaluation, usability, and outcomes (impact layer) and dissemination and acceptance (people layer).

- This is followed by the behavioral responsibility (ethics) sector (our ethical and social responsibility).
Components within this sector include: ethics (science layer); legal/ regulation (application layer); socioeconomic (impact layer) and personal responsibility (people layer).

- The next sector is education (best practices) (educating ourselves and others). This sector unfolds as: health informaticians (science layer); implementers (application layer); health professionals (impact layer) and people education (people layer).

- The next sector represents the multiple types of relationships(our responsibility to build relationships among stakeholders). Within this sector are the following: academic (science layer); professional (application layer); governments/agencies (impact layer); and citizen organizations (people layer).

- The final sector is the connectivity of IMIA (our obligation to share, distribute and disseminate). The components within this sector include: global (science layer); regions (application layer); nations (impact layer); and individuals (people layer).

\section{Health}

The most important mandate for IMIA is to contribute, through the use of Information and Communication Tech-

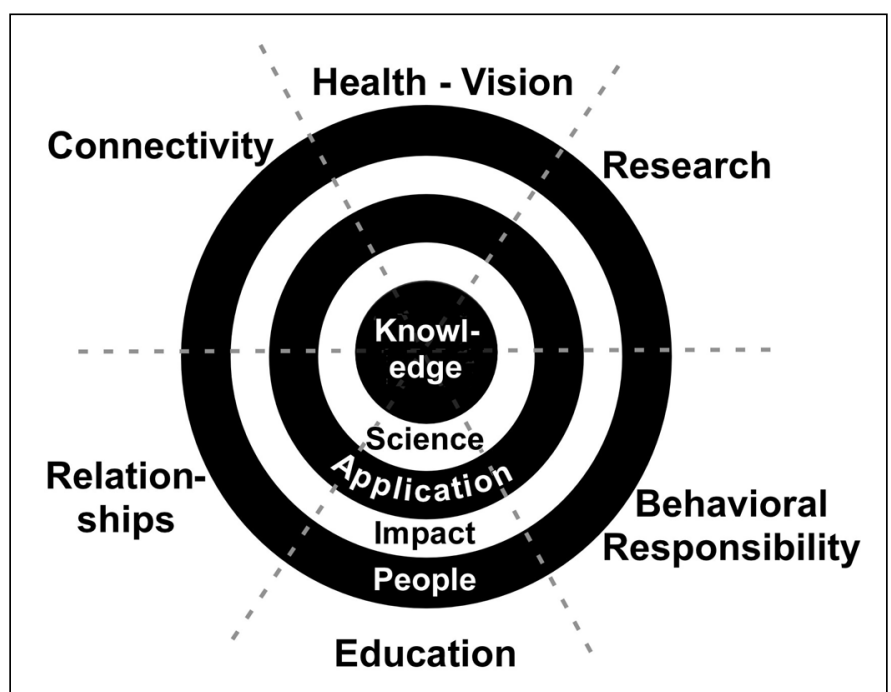

Fig.2

The five circles and six sectors of the IMIA Integrated Strategic Planning Framework 
nology (ICT), to the improvement of biomedical research, clinical practice and public health. It is envisioned that, in the coming years, a more complete knowledge of the different factors that contribute to the development of disease (genetics, environment) will be increasingly available for developing new preventive, diagnostic and therapeutic solutions. IMIA has a key role to play in anticipating new challenges for informatics in the most important trends of future medicine (regenerative, genomic, longevity, patient-centred, preventive). In this regard, working groups will have to demonstrate their leadership and provide guidance to the whole community. The following is a general description of these challenges in accord with the IMIA strategic planning framework model.

New data arises daily from technological advances in biology (e.g. genomics, proteomics) coupled with the wealth of clinical data existing in healthcare settings. This data can be used to decipher the intricate causes of disease, the effects of human genetic variation and its interplay with environmental risk factors and life habits. This information is represented in the form of dynamic pathways, genetic networks, interaction maps and others, in an attempt to capture the bio-psycho-social underlying complex diseases. Modelling and simulation of multi-level entities from molecule to population will allow explanations of observations to predict the effects of external stimuli on the systems. These fields are not traditionally within the umbrella of health informatics; however, their incorporation will provide many future opportunities for biomedical informatics.

In terms of health care, the most important challenge for biomedical informatics is to facilitate a fast and reliable translation of the biomedical research findings into real-use clinical solutions. From this perspective a ma- jor goal is to facilitate "translational research", improving the diagnostic arsenal with new imaging systems and micro devices suitable for "point of care" solutions, promoting rational drug design and supporting the development of personalized therapeutic strategies that can guarantee efficacy and patient safety. New experimental approaches (cell therapy, tissue engineering) raise important issues to be addressed by ICT in health. Informatics can substantially contribute to the advance of these fields. This knowledge must be applied not only at an individual level, in terms of patient care, but also to improve the health of populations, through new public health studies and programs. The aggregation of electronic health records and informatics infrastructures to facilitate longitudinal and bio-bank-based association studies poses new opportunities for health informaticians.

Finally, once validated, this knowledge has to permeate to the individual, who will be in a better position to participate in the maintenance of their integrated health record and to make informed decisions about their lifestyles. The contribution of ICT to an individual's health will demand new imaginative methods and systems from informatics.

\section{Research}

Biomedical informatics is a multi-disciplinary area that involves multiple content areas. It is one of the fastest growing subject/content areas in the world. The use of informatics is expected to enhance research efforts in areas such as genomics and proteomics, for example, and also to change the way medicine is practised in the 21 st century. Research in informatics ranges from the theoretical to applied efforts. The demand for more research in biomedical informatics and for biomedical informatics to support other researchers escalates daily.
The US National Library of Medicine (NLM) defines biomedical informatics as the intersection of basic informational and computing sciences with an application domain in health care and biomedicine. The NLM focuses on characterization, management and efficient utilization of data, information and knowledge in health care and basic biomedical sciences. In clinical medicine, health services administration, education and basic biomedical sciences, computers and networks are fundamental tools of discovery, learning, decision making and management. Informatics research is interdisciplinary and employs a range of research methodologies. The techniques may include quantitative or qualitative approaches, including laboratory and field studies, surveys and needs analyses, modeling and simulation studies. There are a number of research themes that our colleagues throughout the world are studying, including: information and knowledge processing, storing very large datasets, knowledge representation, usability, and management and utilization of information.

Knowledge is the core for IMIA's existence. At the heart of our knowledge based organization is basic core research all of the domains of biomedical informatics. The majority of the domains were outlined in 2001 in the IMIA Scientific Map [7]. Basic research in the topics listed in the Scientific Map is needed throughout the informatics world. IMIA's role will be to stimulate and connect researchers to enable their research.

In addition to the basic core research of informatics, there is an applied science informatics that is the translation between the research and the application of that research to the implementation of many informatics initiatives. Applications could be in the form of products, services, methods, and consultations to name but a few. Informatics products, services, and methods 
have outcomes that will be beneficial for individuals, organizations, research, policy, and so forth. It is imperative that informatics professionals conduct evaluation and test the outcomes (usability) of informatics research, products, and/or services.

For a discipline to thrive and grow it must disseminate the results its basic core and applied research as well as the results of its evaluations and outcomes. Once the information is disseminated through multiple sources, then the results will allow for a greater acceptance of the outcomes of the discipline of biomedical informatics.

\section{Behavioral Responsibility}

IMIA has a responsibility to ensure that it develops, utilizes and shares the knowledge that it possesses responsibly. IMIA has a responsibility to ensure its members and through them the health of citizens of the world, benefit from this knowledge. Appropriate behavioral responsibility can support nations; organizations, communities and individuals in getting the right health information in the right format, to the right person, at the right time.

The core science of IMIA's behavioral responsibility is the underlying approach to its activities, which is based on ethical rights and duties. IMIA promotes these rights and duties through its health informatics ethics principles including privacy and disposition, openness, security, access, legitimate infringement, least intrusive alternative and accountability.

Ethical rights and duties provide a foundation for various legal rights and duties that guide the application these ethical principles. The law provides the minimum standard as envisioned by legislators, judges and juries. These laws govern confidentiality, security and access to health information and how it is obtained, used and disclosed. There is a differentiation between personal health information, where the emphasis is on security and confidentiality, and general information about health strategies and services, where an open approach is promoted.

A range of social and economic factors will influence the impact of these ethical principles and legislative framework on health outcomes. Social factors that may include concepts of health/illness, disease patterns and economic factors are influenced by population structures and our ability to meet the needs of the population through new health technology and knowledge. For example, an individual social status will influence health, their level of knowledge and ability to improve their health by accessing information and heath care. The economic status of each country will determine the degree of development and priorities in health services it provides and public or consumer accountability has emphasized the need for an efficient and effective health services and ensuring value for money. IMIA can promote an ethical approach, within the context of these social economic factors. Informaticians have personal responsibility to ensure that they are guided by IMIA's ethical principles as they engage in their various responsibilities.

\section{Education}

IMIA's raison d'etre is knowledge, and education is the major means of knowledge transfer. IMIA has numerous roles to play in providing leadership in the development and delivery of education, and where appropriate through collaborating with other organizations in contributing to the development of education across the entire spectrum of people impacted by informatics. This includes academics and researchers who develop and disseminate knowledge, informatics professionals who develop and imple- ment knowledge-based tools, health care professionals who use knowledgebased tools, and individual citizens who use health related knowledge for their personal benefit. In this context, the word 'education' is used for all forms of education, training and professional development, although the precise mix of each, and the role that IMIA plays, will vary according to the group or content. Through the efforts of its Health and Medical Informatics Education Working Group, IMIA developed a set of international recommendations for health and biomedical informatics education programs and courses. [8] These recommendations are dynamic and will be continually updated as the nature and scope of health and biomedical informatics knowledge evolve. The recommendations will provide guidance for the development of education at all academic levels for health informaticians, plus people involved in implementation of health information systems, health professionals, and the wider public. IMIA's direct role in education relates to education for biomedical informaticians, but it also has important indirect roles in ensuring the highest quality of education for all other groups of individuals who have a direct or indirect interaction with the practice of biomedical informatics.

IMIA will collaborate with others regarding the accreditation of health and biomedical informatics education programs, courses or subjects and with other bodies regarding certification and registration of biomedical informatics professionals. IMIA's goal is to promote the highest professional standards in biomedical informatics practice, based in the highest education standards and appropriate competencies.

IMIA's Academic Institutional members are the world's leading providers of biomedical informatics education and they continuously ensure that core biomedi- 
cal informatics knowledge is applied within their respective curricula. Most of IMIA's Academic Institutional members are active in research within health and biomedical informatics and also for pedagogical development. They ensure that leading edge research developments are included within their curricula.

IMIA's Academic Institutional members recognize the importance of collaboration and co-operation, among themselves, with other IMIA members and other organizations. 'Connections and collaboration' provides a framework for joint projects, collaboration and exchanges of ideas, information, resources and people for the development of health and biomedical informatics education. Parts of this collaboration include the development and sharing of repositories or directories of information, health informatics literature, expertise and educational programmes. They are developing explicit links to support networking and collaboration, so as to foster dissemination of knowledge, and a range of options for collaboration in education and research.

IMIA has a role in developing educational and research opportunities within biomedical informatics, and mechanisms for fostering the development of 'the next generation' and discovering the currently unrecognised and underdeveloped talent of existing biomedical informatics students.

People and organizations involved in the development and implementation of health information systems will also be influenced by IMIA's educational roles and responsibilities to ensure that systems are developed that meet the needs of the health sector. All health professionals will increasingly use health information systems, and thus their roles will involve an element of health informatics practice. IMIA will encourage those responsible for the curricula of health professionals and their con- tinuing professional development to incorporate the information from the IMIA knowledge core and best practice information into their education efforts.

IMIA has an obligation to ensure that individual citizens benefit from biomedical informatics to improve their health and healthcare. This includes educating citizens about the knowledge and skills to access relevant health information and how biomedical informatics applications can improve their health. Through relevant members and other bodies such as IMIA's Working Groups, IMIA will work with other organizations that seek to encourage the dissemination of educational opportunities to citizens.

IMIA will support the development of education, training and continuing processional development for everyone involved in, or touched by, biomedical informatics by working towards the development of the best content, exemplary pedagogical practice and processes, and contributing to the exploration and integration of appropriate educational technologies.

\section{Relationships}

IMIA has a responsibility to ensure productive and progressive growth in global health by building relationships among the various stakeholders. Biomedical informatics is a dynamic field requiring continuous interaction among various interests. Basic science, research and education are essential to any field. However, application is required to improve global health by effecting workflow, communication, security and utility. Effective bi-directional relationships can support collaboration and true applicability of biomedical informatics. IMIA is the substrate to encourage growth and interaction among academic, professional, corporate, governmental and community-based citizen organizations.
IMIA membership includes six categories of organizations - academic institutions, corporations (health information technology providers, consultants and publishers), national societies for biomedical informatics, correspondents from countries without eligible national organizations and affiliate international agencies. (e.g. World Health Organization) Representatives from each come from varied backgrounds. One of IMIA's core values is its encouragement and nurturing of relationships among the varied interests: education and learning, the business and workflow of healthcare delivery, governmental concerns of population management, and citizen group concerns for quality, safety and security.

Academic organizations gain significantly from IMIA's collaborative atmosphere and its working and special interest groups. Understanding the application of new technologies and creating new technological advances, academic institutions test, modify and expand existing knowledge. Academic organizations also provide a worldwide network for the education and training of existing and new generations of medical informatics professionals.

The business side of biomedical informatics, member corporations of IMIA, also expands on new technology development and applications. In concert with practicing clinical care professionals_academic institutions, these organizations manage the practical application of new and existing biomedical informatics technology. Coordinating clinical knowledge within individual and organizational workflow and proving effectiveness and outcomes requires significant collaboration on methodology, operations and measurement. IMIA provides the infrastructure for discussion and growth on a global basis, enabling more rapid growth of biomedical informatics applicability to actual clinical care efforts. 
Clinical care professionals provide healthcare services based on knowledge. However, enhancement of that knowledge to determine how it best applies to groups and populations of individuals is the purview of governments, ministries of health and local, regional and global agencies. IMIA provides the infrastructure and stimulus for partnerships among academic, professional and governmental organizations to encourage and enhance information flow to manage the world population health bi-directionally.

Citizen organizations and groups focus on individual needs with respect to local and cultural distinction. IMIA provides the environment through which local groups can identify new or alternative means for healthcare delivery and education enabled through informatics. Examples include telecommunication solutions for disadvantaged and inaccessible locations and populations. It is through engendering relationships, networking and communication that IMIA can lead improvements in global health. Academic, professional, governmental and citizen-based organizations are all key stakeholders in global health. IMIA provides the infrastructure to strengthen the relationships among these groups, through informatics, leading the way to improvements in health and healthcare.

\section{Connectivity}

IMIA believes that its members have a duty and obligation to share, distribute and disseminate knowledge to the world through its infrastructure and membership - in order to reach the ultimate beneficiary of the application of knowledge, the individual citizen. IMIA shares knowledge globally through the activities of its working and special interest groups, the published contributions of individual scientists and researchers in scholarly publications, and through its World Congresses (Medinfo).
IMIA enhances the access to knowledge by the nations of the world through the promotion of interaction among and between its member constituencies that represent national, academic, corporate and other infra-structural interests in the world community, as well as the scientific and academic domains that constitute them and the Working and Special Interest Group's fields of scientific knowledge and expertise. IMIA disseminates knowledge to regions of the world through its formal regional structures, EFMI, APAMI, HELINA, IMIA-LAC, and others yet to be formalized. Through its academic members, affiliations with other related organizations and knowledge leaders within its membership, it fosters supports and nurtures the growth and maturation of scientific and educational communities within developing areas of the world.

Through this open and transparent sharing and dissemination of knowledge by the global community, IMIA aims to assist in facilitating the access of the individual to information conducive to the maintenance and betterment of personal health.

\section{Summary}

The IMIA Conceptual Strategic Planning Framework stresses the following:

- IMIA aims to improve biomedical research, clinical practice and public health (VISION)

- IMIA aims to support investigation and development of advanced information systems and technologies (RESEARCH)

- IMIA aims that its efforts are carried out in accordance with strict ethical and legal rules (BEHAVIORAL RESPONSIBILITY)

- IMIA aims to promote education for and about biomedical informatics (EDUCATION)

- IMIA aims to bridge relevant internal and external groups and organi- zations (RELATIONSHIP)

- IMIA aims to incorporate multiple individuals, groups and organizations to constitute the IMIA Association (REACH)

The Conceptual Framework for IMIA's strategic plan is comprehensive and demonstrates the multiple connections and interactions that are possible. This framework provides IMIA with an excellent opportunity to focus its plans to ensure that highest probability of success is possible.

\section{Strategic Goals}

The IMIA Strategic Planning Task Force selected 2015 as the target year. While many of the goals will be met long before 2015, it appeared necessary to select a distant date for the more complex goals. The following represents the model selected to illustrate our planning direction; for each Strategic Goal, several planned or possible projects to meet the goal are summarised. These are illustrative, and not exclusive, and where work is not already under way, specific proposals to undertake the projects will be sought.

\section{IMIA's Core: Knowledge}

Knowledge is at the centre of all IMIA activities and interests. It is the first dimension of IMIA and it is represented as the central core from which all the concentric circles and sectors of IMIA's Integrated Strategic Framework Model originate emanate.

IMIA is the scientific informatics association through which the world's knowledge leaders come together to create, assemble, integrate, synthesize or assimilate the intellectual knowledge that is required worldwide to advance biomedical informatics in its role of improving health and healthcare. It is vital that IMIA provides an indication of the nature and 
scope of this core knowledge, and that the knowledge core encapsulates the current domain as well as providing scope for envisioning the future domain.

\section{The Knowledge Goal}

IMIA will update the 2001 IMIA Scientific Map to reflect the expanding core knowledge of health and biomedical informatics and will ensure that the knowledge map remains up to date. The IMIA Scientific Content Map [7] provided a first overview of the biomedical informatics domain. In any rapidly changing field of knowledge, it is vital to that a current overview of knowledge be updated frequently. A project between IMIA, the British Computer Society Health Informatics Forum (BCSHIF) and CHIRAD (Centre for Health Informatics Research and Development) is updating the IMIA Scientific Content Map, through a review of the literature using electronic tools developed since the first version. This work builds on past BCSHIF and CHIRAD activities and will use multiple methods (literature review, document analysis, and world-wide content expert opinion) to obtain different perspectives. The results will be available for discussion and refinement by the international community before final agreement through a consensus event or activity.

\section{Strategic Sector Goals}

\section{Health Strategic Goal}

IMIA will provide advice, information resources and guidance on how health and biomedical informatics can best support new trends in medicine and healthcare (regenerative, personalized, nanomedicine) from the perspective of potential impact on populations, clinical settings and personal health. This will be provided through the IMIA com- munity (SIGs, WGs, academic and corporate members and national networks.

\section{Projects}

- Surveillance: To outline strategies for an easy to use, widely available bio-surveillance strategy. A Task force has been created.

- Personal Health Records: Collaborate with national and regional efforts, e.g. TET Traveler's Health Record Project (APAMI).

- Analysis of Challenges: Produce a white paper outlining new opportunities for biomedical informatics in the context of genomic, regenerative or nanomedicine technologies.

\section{Research and Science Strategic Goals}

IMIA by 2015 will be the authoritative source for internationally stimulating, organizing and recognizing research contributions, challenges, directions and ethical principles in biomedical and health informatics research. IMIA will be a scientifically independent international sponsoring body for collaborative and competitive activities to further excellence in health and biomedical informatics research.

\section{Projects}

- Establish IMIA competitions/awards.

- Establish a database to facilitate international collaboration opportunities, and to network complementary researchers to work together towards addressing grand challenges in biomedical informatics.

- Publications:

- Update the IMIA Yearbook with sub fields of biomedical informatics as established by the knowledge map.

- Create a new IMIA Magazine for popular reports on projects.

- Establish global connections to facilitate and improve informatics research methods. These include creating a repository of research groups in biomedical informatics topics; a web page of URLs; a research wiki with sub fields and resources for worldwide use.

\section{Behavioral Responsibility Strategic Goal}

Building on the IMIA Code of Ethics, by 2015 , IMIA will be known as the source of best ethical practices in the health and biomedical informatics area.

\section{Projects}

- Develop a comprehensive database of ethics best practice examples, including ethical strategies and potential ethical resource people throughout the world.

- Develop a white paper (with recommendations) regarding the ethical component of information governance worldwide.

\section{Education Strategic Goals}

By 2015 health and biomedical informatics will be a core component of each health-related education program worldwide. [Note: this is targeted at the clinical specialists.] IMIA will be the source for sample informatics curricula that meet required content needs.

By 2015 IMIA will be known as the source health and biomedical informatics education and training program content materials for informatics professionals. By 2015 IMIA will facilitate the connection of non-degree education programs, e.g. this could be continuing education or general introductory education.

\section{Projects}

- Outline a curriculum for the informatics professional who will in turn be responsible to assist the educational systems within their organizations to re-engineer or incorporate informatics into their education program. 
- Create models for potential informatics educational curricula based on the IMIA Knowledge Map and Competencies Map, by providing an updated set of recommendations on health and biomedical education based in those originally developed by the IMIA Education WG. (A Task force is undertaking this work)

- Map informatics competencies based on the IMIA Knowledge Map.

- Endorse the certification process of others bases on the IMA Knowledge Map and competencies map.

\section{Relationship Strategic Goal}

By 2015, IMIA will be the main partner of UN Agencies for achieving ehealth related (millennium) development goals. IMIA will be the reference point for international professional organizations and corporations and it will be the e-Agora for academic exchanges in our field. (An agora was a marketplace or place of assembly in ancient Greece. In modern terms it can refer to a crossroads; metaphorically a crossroads of related concepts, or literally, a point at which physical paths meet).

\section{Projects}

- Create and build the relationship with the UN agencies at the strategic level and with the ability to mobilize the IMIA community

- Use projects elsewhere within this plan to become the e-Agora for academic exchanges in our field.

\section{IMIA as an Organization (Connectivity) Strategic Goals}

IMIA will be recognized as an inclusive global association that connects groups and individuals at the leading edge of the state of the art of the field of health and biomedical informatics, within and without the IMIA community, in order to advance, develop and disseminate the (art and) science of informatics in support of health.

To increase IMIA's global visibility as the association that is capable of connecting the brightest and the best of the world for developing the science of informatics in support of health.

The connectivity will be supported by developing up-to-date legal/operational frameworks, together with appropriate bylaws, policies etc. that are both flexible and provide future-proofed governance models. This might necessitate Board reorganization (roles and responsibilities), plus possible reorganization of groups within IMIA. Outstanding communications (in all senses), through web services/collaborative tools, newsletters, conferences, etc. will need to be further developed, together with strategies to facilitate effective/efficient utilization of IMIA resources.

\section{Projects}

- Revision of existing IMIA bylaws as well as the resultant policies and procedures etc.

- Develop professional expertise resource index.

\section{Implementation}

The motto for the implementation is "let a thousand flowers bloom!" The IMIA General Assemblies in 2005 and 2006 participated in this strategic planning process, and after the 2007 General Assembly meeting, IMIA will engage working groups, national member societies and regions to participate by either developing their goals (working groups) in accord with the strategic plan or by selecting a part of the plan to implement on behalf of informatics world-wide. IMIA has approved the use of a portion of the IMIA reserve funds to fund targeted projects in each of the sectors. The IMIA money will be a token amount that could help a person or organization develop the project deliverable, give it to IMIA and then be prepared to update it or to develop a process for IMIA to update.

\section{Evaluation}

The evaluation goal is to present a yearly update to the IMIA General Assembly of the status of the goals and objectives created and to offer a comprehensive evaluation to the General Assembly that meets in conjunction with the Medinfo conferences.

\section{References}

1. Lorenzi NM. The IMIA Possibility Framework. Methods Inf Med 2004; 3:207.

2. Lorenzi N. Strategy in a fishbowl: an invitation to determine the shape of IMIA in 2015. Methods Inf Med 2006;45:235-9.

3. Lorenzi N. Towards IMIA 2015 - the IMIA Strategic Plan. In: IMIA Yearbook of Medical Informatics 2007. Methods Inf Med 2007; 46 Suppl 1:1-5.

4. The IMIA Strategic Plan: Towards IMIA2015. Available online at http://www.imia.org/images/ IMIA_Strategic_Plan.pdf accessed 03 January, 2008

5. Cabinet Office. Transformational Government: Enabled by Technology. 2005. London: The Stationery Office. Available online at http:// www.cio.gov.uk/transformational_government/ strategy/Last accessed 03 January, 2008

6. The IMIA Code of Ethics for Health Information Professionals. Available online at http:// www.imia.org/code_of_ethics.html Last accessed 03 January, 2008

7. The IMIA Scientific Map. Available online at http:/ /www.imia.org/2002_scientific_map.html Last accessed 03 January, 2008

8. Recommendations of the International Medical Informatics Association (IMIA) on Education in Health and Medical Informatics. Available online at http://www.imia.org/pubdocs/rec_english.pdf Last accessed 07 January, 2008

Correspondence to:

Dr. Peter J. Murray

IMIA Interim Vice President for Strategic Planning Implementation

Coachman's Cottage

Nocton Hall, Nocton, Lincoln

United Kingdom

E-mail: peterimurray@gmail.com 